\title{
Transurethral Foley catheter misplacement into the upper urinary tract in a patient with a history of lung cancer and chemotherapy: a case report and considerations to keep in mind
}

\author{
Soo Kyung Cho ${ }^{1 \#}$, Myung Soo Kim ${ }^{2 \#}$, Ho Seok Chung ${ }^{2}$, Eu Chang Hwang ${ }^{2}$, Seung Il Jung ${ }^{2}$, \\ Dongdeuk Kwon ${ }^{2}$, Kwangsung Park ${ }^{2}$ \\ ${ }^{1}$ Department of Internal Medicine, Kwangju Christian Hospital, Gwangju, Korea; ${ }^{2}$ Department of Urology, Chonnam National University Medical \\ School, Gwangju, Korea \\ \#These authors contributed equally to this work. \\ Correspondence to: Ho Seok Chung, MD, PhD. Department of Urology, Chonnam National University Hwasun Hospital, 322 Seoyang-ro, Hwasun- \\ eup, Hwasun-gun, Jeonnam, 58128, Republic of Korea. Email: hschung615@gmail.com.
}

\begin{abstract}
Indwelling urethral catheter placement is a common and comparatively safe procedure. Misplacement of a urethral catheter into the upper urinary tract is unusual, and only a few cases have been reported. We describe the case of a 43-year-old man who presented with oliguria and had a history of chemotherapy for known metastatic lung cancer. As he had no history of urological disease, urethral catheterization was expected to be uneventful. The catheter was unable to be pulled back to the bladder neck once the balloon was inflated, and the patient expressed discomfort. Subsequent computed tomography revealed that the tip of the catheter was placed in the middle of the right ureter. Unbeknownst to the physicians before urethral catheterization, the patient had severe lower urinary tract symptoms and urinary bladder dysfunction with hydronephrosis, likely due to chemotherapy. Based on the patient's symptoms and imaging results, we judged the possibility of severe ureteral injury to be low. The malpositioned catheter was removed uneventfully after complete balloon deflation and then reinserted properly. He was admitted to the medical department but died as a result of an exacerbation of the underlying disease unrelated to the incident. If urethral catheter placement seems abnormal, physicians should aspirate and irrigate to confirm correct positioning before balloon inflation; then, they should carefully pull the inflated balloon near the neck of the bladder while monitoring the patient's symptoms. Although urethral catheter placement is comparatively safe, physicians must keep in mind that patients who have undergone chemotherapy might be at a risk for this rare complication.
\end{abstract}

Keywords: Complications; drainage; ureter; urinary catheters; case report

Submitted Jun 19, 2020. Accepted for publication Dec 28, 2020.

doi: $10.21037 /$ tau-20-1016

View this article at: http://dx.doi.org/10.21037/tau-20-1016

\section{Introduction}

Indwelling urethral catheter placement is a common and comparatively safe procedure performed in many clinical situations. Unintentional misplacement of the urethral catheter into the upper urinary tract has seldom been reported in the medical literature (1-4). The mechanism of such misplacement is unclear, but it can lead to complications such as ureteral obstruction and ureteral injury. If the patient has no history of urologic disease, physicians are more likely to neglect precautions against malpositioning of the urethral catheter. Herein, we describe a case of urethral catheter placement into the right ureter of a male patient with no history of urologic disease. We present this article in accordance with the CARE reporting 

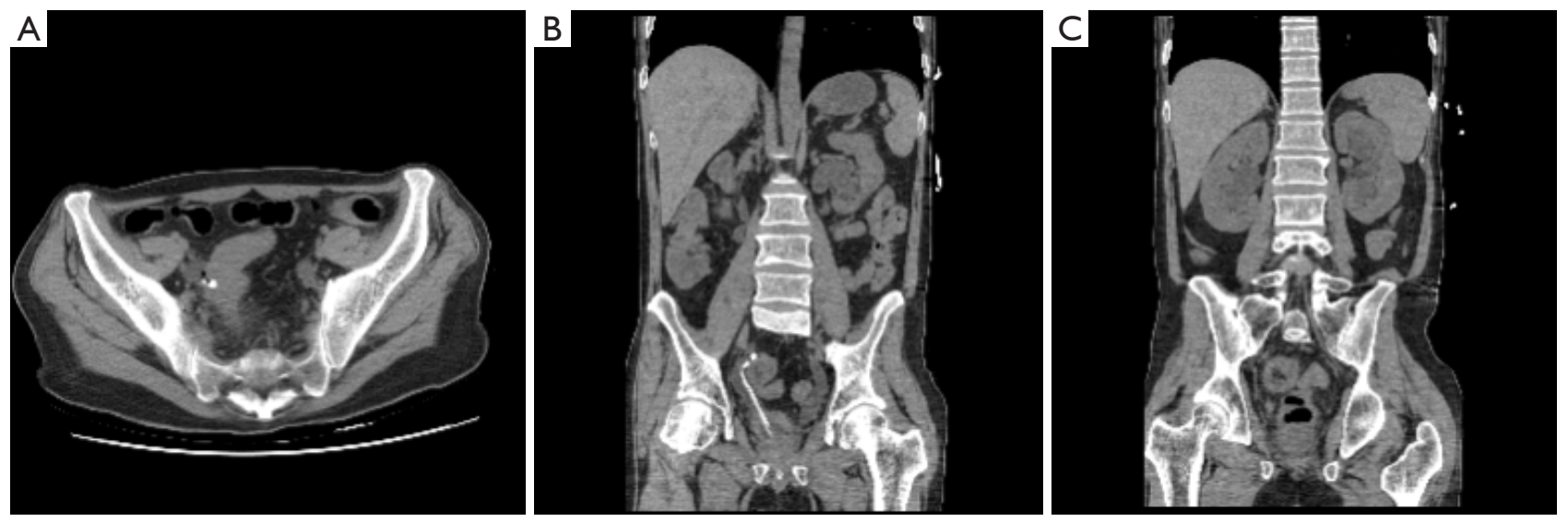

Figure 1 Abdominopelvic computed tomography images. (A) Axial view showing the tip of the urethral catheter placed in the middle of the right ureter with inadequate balloon deflation. (B) Coronal view showing the urethral catheter placed in the middle of the right ureter. (C) Bilateral hydronephrosis.

checklist (available at http://dx.doi.org/10.21037/tau-201016).

\section{Case presentation}

A 43-year-old man presented to our emergency department because of oliguria and general weakness that had started 1 week earlier. He had no history of urologic disease; however, he had a history of metastatic small-cell lung cancer, which had been treated at another hospital with chemotherapy using carboplatin and etoposide (first-line), followed by topotecan (second-line). The previous imaging examinations were not available. The primary physician inserted a 16-Fr Foley catheter for further evaluation and urine output monitoring, which was accomplished without difficulty and a small amount of urine returned. The catheter was unable to be pulled back to the bladder neck once the foley balloon was inflated, and the patient expressed discomfort. In response, the primary physician consulted with the urology department. Catheter irrigation was found to be insufficient, causing right-flank discomfort. Furthermore, resistance was felt during the removal of the catheter after balloon deflation and cutting the side arm of balloon port. To check catheter positioning, we performed abdominopelvic computed tomography (CT). This revealed that the tip of the catheter was placed in the middle of the right ureter; balloon deflation appeared to be inadequate, and moderate bilateral hydronephrosis was also observed (Figure 1). However, as no definite signs of gross hematuria on a Foley catheter or periureteral fluid collection on CT scan, the possibility of severe ureteral injury appeared low. Subsequently, after complete balloon deflation, the malpositioned catheter was removed uneventfully and reinserted properly. The remainder of the patient's hospitalization was uneventful, and ultrasonography performed 3 days later showed that the catheter was located in the right place, with no definite abnormal findings except mild bilateral hydronephrosis. The patient requested medication for voiding dysfunction but refused further urological evaluation and urethral catheterization. One month later, he presented again with a poor general condition, and follow-up CT revealed aggravated bilateral hydronephrosis (Figure 2). He was admitted to the medical department, and urethral catheterization was performed; however, his condition terminated in death of multi-organ failure related to cancer progression after 2 weeks. The timeline of this case is shown in Figure 3. All procedures performed in studies involving human participants were in accordance with the Declaration of Helsinki (as revised in 2013). Written informed consent was obtained from the patient for the publication of this case report and any accompanying images.

\section{Discussion}

Indwelling urethral catheter placement is a widely performed clinical procedure and is considered comparatively safe. Serious complications rarely occur, with the most common being infection, urethral injury, catheter malfunction, and catheter balloon inflation in the urethra (5). 

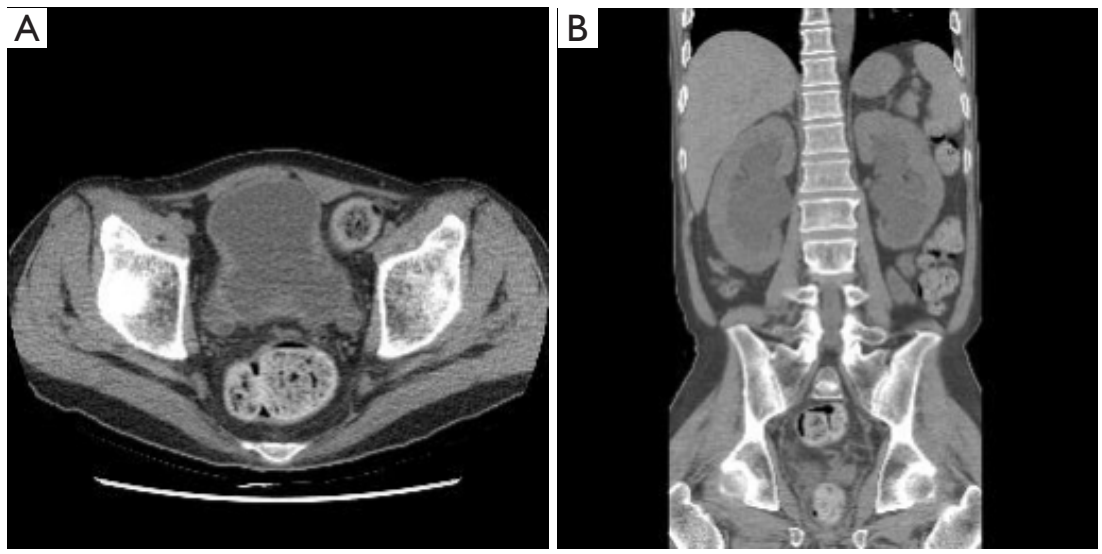

Figure 2 Follow-up abdominopelvic computed tomography images showing (A) a thick-walled bladder (B) with aggravated bilateral hydronephrosis.

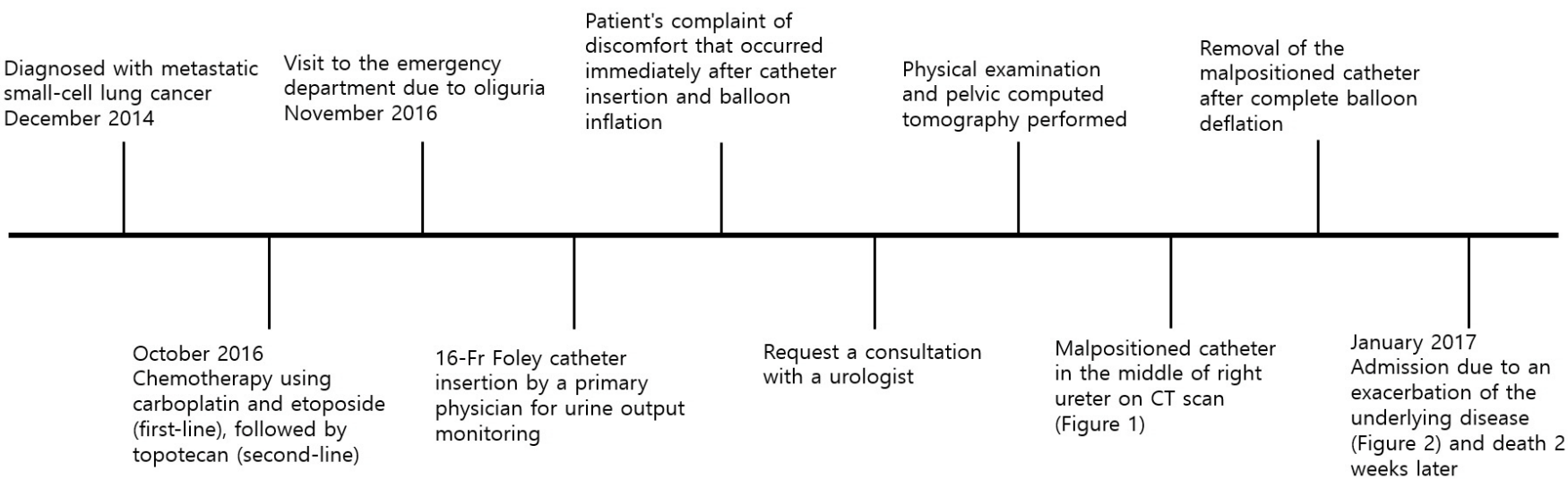

Figure 3 The timeline of this case.

Unintentional catheter misplacement into the upper urinary tract has rarely been reported, with only 17 cases described in the literature (1-4).

Ishikawa et al. (1) suggested female sex, neurogenic bladder, and sensory disorder as major risk factors for misplacement of an indwelling urethral catheter into the ureter. The female urethra is short enough for the catheter to enter the ureter easily owing to misdirection, and neurogenic bladder is a condition that requires chronic urinary catheterization; meanwhile, a sensory disorder may result in the patient experiencing no discomfort or pain during catheter misplacement. In addition, urethral catheterization with an empty bladder may be a risk factor for ureteral placement (6). If catheter misplacement is suspected, a diagnosis can be made using ultrasonography, CT, and cystoscopy (1-4).
However, our patient was male and had no history of urologic disease or sensory disorder; thus, there was no obvious risk factor for catheter misplacement. Abdominopelvic CT revealed bilateral hydronephrosis, suggesting an unexamined chronic voiding disorder linked to his lung cancer treatment. However, this was difficult to predict before the imaging study. Chemotherapy-induced neuropathy can cause bladder dysfunction (7). Also, cancer progression with a poor general condition might be related to neurogenic bladder dysfunction with hydronephrosis in this case.

As in our case, urethral catheter misplacement into the ureter may lead to the return a small amount of urine in patients with hydronephrosis. Positioning of the catheter before balloon inflation is a basic and important procedure. The location could be estimated by the flow 
of urine through the inserted catheter, but in oliguric and anuric patients, confirmation through irrigation and fluid inhalation is more effective $(8,9)$. In other words, if there is any abnormality in the catheter placement, the catheter must be aspirated and irrigated to confirm the correct position before inflating the balloon.

The removal of the catheter is deflation using a conventional manual syringe aspiration. In particular, very slow deflation or passive deflation is appropriate to minimize ballon cuffing or catheter entrapment. If these methods do not work, cutting off the inflation valve or direct percutaneous puncture could be considered. However, it is important to keep in mind that it is essential to sufficient exploration for the cause by image study prior to removal, and real-time manipulation under image guidance using an ultrasound device or fluoroscopy could be helpful in some cases.

Our patient was managed nonoperatively and without ureteral stenting because no definite signs of severe ureteral injury were observed on CT. This lack of injury might be related to the patient's hydronephrosis. In previous similar cases, a patient with bilateral hydronephrosis was spared ureteral injury; this was also observed in another patient with a patulous ureter with vesicoureteral reflux $(10,11)$. If a ureteral injury is detected, management requires ureteral stent insertion or surgery according to the type of injury and time until detection, and the patient should be monitored for the possibility of complications such as ureteral stricture (12).

Our patient had a history of lung cancer and chemotherapy; thus, similar situations might occur in other cancer patients. Furthermore, our case findings suggest that urethral catheter malpositioning can be treated nonoperatively if it does not cause severe injury. In this case, patient death prevented us from assessing the potential long-term complications arising from catheter malpositioning. However, after several days of follow-up, we observed no definite related symptoms and no abnormal ultrasonographic findings.

The present case report describes an unusual complication during urethral catheterization that physicians may encounter in clinical practice. To prevent such situations, physicians should always keep in mind the possibility of catheter misplacement into the upper urinary tract, particularly in patients with risk factors such as female sex, neurogenic bladder, sensory disorder, empty bladder, or a history of chemotherapy. If adequate urine volume is not drained after catheter insertion, catheter irrigation should be performed before balloon inflation. If the urethral catheter is not in the proper position, balloon inflation is likely to cause pain, unless the patient has a sensory disorder.

\section{Conclusions}

Physicians should keep in mind that urethral catheter misplacement into the upper urinary tract can occur even in patients with no definite risk factors. To avoid such complications, physicians should take care to follow the basic principles of catheterization, even if the procedure is considered simple and easy to perform.

\section{Acknowledgments}

Funding: None.

\section{Footnote}

Reporting Checklist: The authors have completed the CARE reporting checklist. Available at http://dx.doi.org/10.21037/ tau-20-1016

Conflicts of Interest: All authors have completed the ICMJE uniform disclosure form (available at http://dx.doi. org/10.21037/tau-20-1016). The authors have no conflicts of interest to declare.

Ethical Statement: The authors are accountable for all aspects of the work in ensuring that questions related to the accuracy or integrity of any part of the work are appropriately investigated and resolved. All procedures performed in studies involving human participants were in accordance with the Declaration of Helsinki (as revised in 2013). Written informed consent was obtained from the patient for the publication of this case report and any accompanying images.

Open Access Statement: This is an Open Access article distributed in accordance with the Creative Commons Attribution-NonCommercial-NoDerivs 4.0 International License (CC BY-NC-ND 4.0), which permits the noncommercial replication and distribution of the article with the strict proviso that no changes or edits are made and the original work is properly cited (including links to both the formal publication through the relevant DOI and the license). See: https://creativecommons.org/licenses/by-nc-nd/4.0/. 


\section{References}

1. Ishikawa T, Araki M, Hirata T, et al. A rare complication: misdirection of an indwelling urethral catheter into the ureter. Acta Med Okayama 2014;68:47-51.

2. Modi PK, Salmasi AH, Perlmutter MA. Inadvertent foley catheterization of the ureter. Can J Urol 2014;21:7326-29.

3. Bradley A, Sozener C. Incidentally Discovered Foley Catheter Placement Into a Transplanted Kidney. Urology 2015;86:e11-2.

4. Yune JJ, Lightfoot M, Baldwin DD, et al. Unsuccessful Foley catheterization had an unexpected explanation. Female Pelvic Med Reconstr Surg 2015;21:e14-6.

5. St Luce S, Ninan AC, Hall JA, Kimberl IJ, Petros JA, Issa MM. Role of transrectal ultrasonography in diagnosis and treatment of retained Foley catheter. Urology 2005;65:1001.

Cite this article as: Cho SK, Kim MS, Chung HS, Hwang EC, Jung SI, Kwon D, Park K. Transurethral Foley catheter misplacement into the upper urinary tract in a patient with a history of lung cancer and chemotherapy: a case report and considerations to keep in mind. Transl Androl Urol 2021;10(3):1347-1351. doi: 10.21037/tau-20-1016
6. Lowthian P. Aberrant Foley catheter placement into the proximal right ureter. BJU Int 2002;90:475-6.

7. Quasthoff S, Hartung HP. Chemotherapy-induced peripheral neuropathy. J Neurol 2002;249:9-17.

8. Daneshmand S, Youssefzadeh D, Skinner EC. Review of techniques to remove a Foley catheter when the balloon does not deflate. Urology 2002;59:127-9.

9. Patterson R, Little B, Tolan J, et al. How to manage a urinary catheter balloon that will not deflate. Int Urol Nephrol 2006;38:57-61.

10. Muneer A, Minhas S, Harrison SC. Aberrant Foley catheter placement into the proximal right ureter. BJU Int 2002;89:795.

11. Kato H. Incorrect positioning of an indwelling urethral catheter in the ureter. Int J Urol 1997;4:417-8.

12. Bryk DJ, Zhao LC. Guideline of guidelines: a review of urological trauma guidelines. BJU Int 2016;117:226-34. 